interest. Again, it is one which Haldane deals with at length in his paper. For the reason stated, I was not aware that he had done so until after my own letter appeared. Levine and his co-workers do not explicitly assert that the combination $R h(-)$ mother and $R h(+)$ foetus constitutes a sufficient, in contradistinction to a necessary, condition for the occurrence of erythroblastosis fotalis; but their writings are certainly open to that interpretation, and I have recently met several eminent clinicians, including two responsible officers of blood transfusion depots, who interpret Levine's hypothesis in the former sense. Now the approximate incidence of $R h(+)$ offspring of $R h(-)$ mothers is easy to calculate. Indeed such calculations appear in preliminary notes of the pioneer work being done by the Galton Laboratory Serum Unit. They show that such offspring are vastly in excess of any recorded statistics of the incidence of neonatal hæmolytic anæmias, even after generous infla. tion of the figures by due allowance for fotal deaths.

There are several possible reasons for this. I have suggested one, and could suggest several others. Haldane ${ }^{4}$ offers the interesting hypothesis that women differ individually with respect to permeability of the placenta. It would therefore be interesting to know if an $R h(-) O$ woman who had a history of miscarriage in a first marriage with an $R h(+) O$ man would sustain the family tradition in a subsequent union with an $R h(-) A$ man. In any event, there seems to be little doubt about the truth of either of two propositions : $(a)$ that the genotype combination of the mother and offspring does not disclose a sufficient condition for the occurrence of erythroblastosis; (b) that it does disclose a necessary condition for a very high percentage of cases.

The distinction, if elementary, has some practical significance at the present moment, because voluntary donors at blood transfusion centres may be misled by popular or semi-popular expositions of Levine's important discovery, including his own in a recent issue of the Journal of Heredity. Indeed, my interest in its genetic implications was first excited by hearing of a Rhesus-negative young woman who had withdrawn her promise or offer of marriage when her donor fiancé proved to be $R h(+)$. I cannot improve on Haldane's own statement of the practical issue in his paper on the adverse form of selection which Levine's hypothesis implies: "it is obviously futile to urge that all $r h . r h$ women, some $14 \%$ of the total, should be prevented or dissuaded from marrying the $R h . R h$ and $R h . r h$ men who make up the remaining $86 \%$. Research on the etiology of high placental permeability is an urgent problem. If it is largely due to a particular gene substitution, the gene in question must be much rarer than $r h$ and therefore a more suitable target for negative eugenics."

With reference to the statement that the Galton Laboratory Serum Unit now postulates seven alleles, of which "not more than four are Rhesus positive", it sulfices to say this. If progress continues at this encouraging tempo, the $R h$ locus may yet prove to be as unstable as I have ventured to suggest.

LaNCELot HogBen.

The University,

Birmingham, I5. Jan. 27.

\footnotetext{
I NATURe, 152, 721 (1943).

2 NATURE, 153, 106 (1944).

3 NATURE, 153, 106 (1944).

Ann. Eug., 11, 333 (1942)
}

\section{Crossing-over in the Males of Drosophila subobscura}

Merosis in the males of the higher Diptera is characterized by absence of chiasmata. This means that as a rule genetical crossing-over occurs only in females. There are, however, a few exceptional families in Drosophila melanogaster ${ }^{1,2}$ and virilis ${ }^{3}$, the constitution of which can only be explained as due to crossing-over in the father. In these cases the exceptional offspring occurred in batches rather than singly. It was therefore supposed that crossing-over had taken place in one of the early spermatogonial divisions, though there is no cytological basis for this assumption.

I have found, in Drosophila subobscura, cytological evidence that crossing-over can take place in males during meiosis. Of three hundred testes from stocks containing 1-3 pairs of large autosomal inversions, one contained a lobule in late first anaphase, in which eight of the thirty-two nuclei in division showed single chromosome bridges, one a bridge and a fragment, and another two bridges. This is evidence that crossing-over had taken place in ten out of thirty-two cell divisions, because single crossing-over in inver. sions which do not include the centromere results in dicentric and acentric fragments. The dicentric fragments form bridges either at the first or second meiotic division, and the acentric fragments are lost.

Thus a male Drosophila still has the possibility of forming chiasmata. Normally, however, the meiotic chromosomes first emerge from a diffuse condition in the diakinesis stage of prophase ; the pachytene stage where chiasmata are formed in a normal meiosis has never been seen. A slight alteration in timing, which affects all the cells in a lobule simultaneously, may permit chiasma formation, thus showing that the properties of the chromosomes have not changed radically. This results in the occasional appearance of a number of cross-over individuals in the progeny of a single heterozygous male.

Ursula Philit.

Biometry Department,

University College, London, at Rothamsted Experimental Station, Harpenden, Herts.

Jan. 25.

${ }^{1}$ Muller, Amer. Nat., 50 (1916).

${ }^{2}$ Friesen, Biol. Zbl., 54 (1934).

${ }^{3}$ Kikkawa, Proc. Imp. Acad. Japan, 9 (1933).

\section{Fluctuations in Seaweed}

RECENTLY, several communications have appeared in NATURE relating to seaweeds, their properties and ușes. It is apparent that their commercial potentialities are much more considerable than has hitherto been realized, and that this is not merely a temporary effect of war needs. Obviously, progress must depend on the continuance of adequate supplies, yet little has been said as to the need for the study of their distribution, conservation and protection in favourable localities.

There are, indeed, two opposing views among those who have any opinion in the matter. The one is a firm belief that supplies are 'inexhaustible'; the other, that they are, like land crops, subject to serious fluctuations unless proper precautions are taken. It may be well to consider how far these views are based on general impressions or founded on fact. 